\title{
Depression treatment and health-related quality of life among adults with diabetes and depression
}

\author{
Ebtihag O. Alenzi ${ }^{1}$ and Usha Sambamoorthi ${ }^{1}$ \\ Ebtihag O. Alenzi: eoalenzi@mix.wvu.edu \\ ${ }^{1}$ Department of Pharmaceutical Systems and Policy, School of Pharmacy, West Virginia \\ University, Morgantown, WV 26506, USA
}

\begin{abstract}
Background-Previous findings regarding depression treatment and its consequences on healthrelated quality of life (HRQoL) of adults with diabetes were inconsistent and targeted certain groups of population. Therefore, there is a critical need to conduct a population-based study that focuses on a general population with diabetes and depression.
\end{abstract}

Objective-The primary aim of this study was to examine the physical and mental HRQoL associated with depression treatment during the follow-up year.

Methods-We adopted a longitudinal design using multiple panels (2005-2011) of the Medical Expenditure Panel Survey to create a baseline year and follow-up year. We included adults with diabetes and depression. We categorized the baseline depression treatment into: (1) antidepressant use only; (2) psychotherapy with or without antidepressants; and (3) no treatment. HRQOL was measured using SF-12 version 2 physical component summary (PCS) and SF-12 mental component summary (MCS) scores during both baseline year and follow-up year. Ordinary least squares (OLS) were used to estimate the association between depression treatment and the HRQoL measures. The OLS regression controlled for predisposing, enabling, need, external environment factors, personal health practices, and baseline HRQoL measures.

Results-After controlling for all the independent variables and the baseline PCS, individuals who received psychotherapy with or without antidepressants had higher PCS scores as compared to those without any treatment for depression (beta $=1.28, p<0.001$ ). Individuals who reported using only antidepressants had lower PCS scores (beta $=-0.54, p<0.001$ ) as compared to those without depression treatment. On the contrary, individuals who reported receiving psychotherapy with or without antidepressants had lower MCS scores as compared to those without depression treatment (beta $=-1.43, p<0.001$ ). Those using only antidepressants had higher MCS scores as compared to those without depression treatment (beta $=0.56, p<0.001$ ).

Conclusion-The associations between depression treatment and the HRQoL varied by the type of depression treatment and the component of the HRQoL measures.

Correspondence to: Ebtihag O. Alenzi, eoalenzi@mix.wvu .edu.

Authors' contributions EA contributed in conceptualization, designing, analysis, and interpretation of data and drafted the manuscript. US contributed to conceptualization, designing, analysis, interpretation of data and revised the manuscript. All authors have given approval of the final version to be published.

Conflict of interest The authors declare that they have no competing interests. 


\section{Keywords}

Diabetes; Depression; HRQoL; Antidepressants; Psychotherapy; Adults MEPS

\section{Background}

The high prevalence of depression and its negative consequences on health-related quality of life (HRQOL) among individuals with diabetes mellitus (DM) have been well established $\left[{ }^{1}{ }^{7}\right]$. Previous research has found that individuals with diabetes and depression reported lower HRQOL than individuals without depression $[2,5,8]$. The randomized clinical trials (RCTs) have demonstrated that depression treatment is associated with improvement in depressive symptoms $\left[{ }^{9}{ }^{11}\right]$. Therefore, it is plausible that depression treatment may improve HRQOL as well.

RCTs that have examined the association between depression treatment and HRQoL among individuals with diabetes have produced mixed findings. A RCT on individuals aged 50-70 years, with diabetes and depression, found that those who received paroxetine treatment had better HRQOL using the Short Form (36) Health Survey (SF-36) scores compared to the placebo group after 3 months of treatment. However, the SF-36 scores of the two groups were not significantly different after 6 months of treatment $\left[{ }^{11}\right]$. Another RCT studied the effect of sertraline among low-income Hispanics and African-Americans with diabetes and depression. This trial found that no significant differences between the treatment group and the placebo group in HRQOL during a 6-month period [ $\left.{ }^{12}\right]$.

With respect to psychotherapy, one study among individuals with a major depressive disorder has shown that combined treatment with escitalopram and cognitive behavioral therapy (CBT) improved some self-reported work functioning outcomes compared with escitalopram alone, but it was not effective in symptom-based outcomes [ $\left.{ }^{13}\right]$. RCTs on Hispanic and Latino individuals with diabetes and depression have found that collaborative care significantly improved HRQoL as compared to usual care $\left[{ }^{14}, 15\right]$.

Thus, the inconsistent findings from these trials and differing definitions of HRQoL make it unclear as to whether depression treatment improves HRQOL. Also, previous studies have a restricted generalizability because they studied certain population groups based on their age $\left[{ }^{11}\right]$ or socioeconomic status $\left[{ }^{12}\right]$, and followed individuals for a short duration of time. Furthermore, most of previous studies were RCTs $\left[{ }^{13}{ }^{15}\right.$ ] which examined the influence of CBT on HRQOL among those with diabetes and depression. None of those studies have examined the impact of CBT on HRQOL among individuals with diabetes and depression in real-world settings. Many individuals with diabetes do not seek treatment for depression; most of the above-mentioned studies compared collaborative care with usual care for depression. Thus, further studies are needed to examine the consequences of untreated depression on HRQoL of individuals with diabetes in real-world practice settings.

Therefore, the primary aim of this study is to examine the association between depression treatment and HRQOL measures of adults with diabetes and depression, comparing them to those who did not report any depression treatment. We hypothesize that among adults with 
diabetes and depression, any treatment for depression will be associated with better physical and mental HRQoL in comparison with adults who had not been treated. For the purposes of this study, we adopted a longitudinal design using data from a nationally representative survey, the Medical Expenditure Panel Survey.

\section{Conceptual framework}

The association between depression treatment and the HRQoL is our primary interest. However, other factors can affect this association. Therefore, we used the expanded Andersen behavioral model to guide in the selection of other independent variables that may affect the HRQoL $\left[{ }^{16}\right]$. This model posits that health outcomes and healthcare services utilization are influenced by: (1) predisposing factors (gender, race, and age); (2) enabling factors (marital status, employment status, education, poverty status, health insurance, and prescription drug coverage); (3) need factors (chronic conditions numbers, and baseline HRQoL); (4) personal health practices (smoking status, body mass index (BMI), and physical activity); and (5) the external environment (metro status).

\section{Methods}

\section{Study design}

We used a retrospective longitudinal study design with a baseline period (1 year) and followup period (1 year).

\section{Data source}

We used data from the household component (HC) of Medical Expenditure Panel Survey (MEPS). The MEPS is a nationally representative survey of the non-institutionalized US population conducted annually $\left[{ }^{17}\right]$. Although MEPS is conducted annually, the survey follows individuals for two complete calendar years. Interviews were conducted five times to minimize recall bias and to collect information for two calendar years, which enables researchers to conduct longitudinal studies $\left[{ }^{18}, 19\right]$. For each individual, detailed information of the respondents' demographic characteristics, medical conditions, treatments, perceived physical and mental health status, HRQoL, healthcare utilization and expenditures are collected $[20,21]$.

For the purposes of the current study, the first year of observation was used as the baseline period and second year as the follow-up period. To gain sufficient sample size, we combined data from six panels: 10 (2005-2006), 11 (2006-2007), 12 (2007-2008), 13 (2008-2009), 14 (2009-2010), and 15 (2010-2011).

\section{Study cohorts}

We included adults aged over 21 years or older with diabetes and depression in the baseline year. The MEPS medical condition files contain data on respondents' chronic diseases selfreported by patients and coded by professional coders to be converted to International Classification of Diseases, Ninth Revision, Clinical Modification (ICD-9-CM) codes. Diabetes and depression were identified using the clinical classification code system that 
maps ICD-9-CM codes into medical conditions [ ${ }^{21}$ ]. Since MEPS does not distinguish between type 1 and type 2 diabetes, we included individuals with diabetes mellitus regardless of its type $\left.{ }^{22}\right]$.

\section{Measures}

Dependent variable-The dependent variable is the HRQoL measured during the followup year. We used the summary scores derived from the second version of the 12-item Short Form Health Survey [SF-12]) to measure the HRQoL. The SF-12 measures eight concepts: physical functioning, role limitations resulting from physical health problems, bodily pain, general health, vitality (energy/fatigue), social functioning, role limitation resulting from emotional problems, and mental health. The SF-12 has two summary scores: the physical component summary (PCS) and the mental component summary (MCS) $\left.{ }^{23}\right]$. We used the MEPS generated PCS and MCS scores to represent physical and mental health components of the HRQoL. The SF-12 summary scores range from 0 to 100, with higher scores representing better self-reported health and therefore better HRQoL. A score of 50 represents the mean score of the general US population mean $\left[{ }^{24}\right]$.

Key independent variable-We measured depression treatment during the baseline. We grouped the depression treatment into three categories: (1) no depression treatment; (2) only antidepressant use; and (3) psychotherapy with or without antidepressants. We identified antidepressants from the prescription medicines events files. Antidepressants were identified using the Multum therapeutic codes available in the prescribed medicines events files. Psychotherapy was identified using the MEPS outpatient visit files [ $\left.{ }^{25}\right]$.

Other independent variables_-All other independent variables were measured during baseline year.

(1) Predisposing factors included gender, race, and age. Race was categorized into white and others (we combined all other race groups because we did not have enough sample size in each group). Age was categorized as 22-49, 50-64, and 65 years or older. (2) Enabling factors were: marital status, education, poverty status, health insurance, and prescribing drug coverage. Marital status was classified as married and unmarried, and education groups were categorized into less than high school, high school graduate, and above high school. Poverty status was defined as poor/near poor [family income $<124 \%$ of the federal poverty line (FPL)], middle income (200-399 \% of FPL), and high income ( $\geq 400 \%$ of FPL). Health insurance groups were private, public, and uninsured, and prescription drug coverage was yes or no. (3) Need factor consisted of the presence or absence of the following chronic conditions: (asthma, arthritis, cancer, gastroesophageal reflux (GERD), thyroid, high cholesterol, hypertension, heart disease, and anxiety). The number of chronic conditions was categorized into three groups ( $\leq, 2-4$, and $\geq 5$ conditions) for bivariate analysis only. (4) Personal health practices were: smoking status, BMI categories, and physical activity. Smoking status were current smoker and others, and BMI categories were underweight/ normal (less than or equal to 24.9), overweight (25-29.9), and obese ( 230 ). Physical activity was categorized into vigorous or moderate activities (at least 3 days a week) and other. (5) 
The external environment consisted of metropolitan status (metropolitan and not metropolitan).

\section{Statistical analysis}

The unadjusted relationships between depression treatment categories and baseline characteristics were tested with Chisquare statistics. The unadjusted relationship between depression treatment categories and the HRQoL measures (PCS and MCS) were tested using $F$ tests. The adjusted associations between depression treatment categories and the HRQOL measures (PCS and MCS) were examined in separate ordinary least squares (OLS) regression models. A series of four OLS regression models were fit. Model 1 included depression treatment categories, predisposing, enabling, and need factors; model 2 additionally included the personal health practices; model 3 included all the factors in model 2 plus the external environment characteristics; and the final model 4 included predisposing, enabling, need factors, personal health practices, the external environment characteristics, and the baseline HRQoL measures. The regression models were compared using $F$ statistics and adjusted $R^{2}$. For simplicity, we only present the results from models 3 and 4 in the paper. All tests were two-sided, and the significant levels were set at 0.05 . We used the survey procedures (SURVEYMEANS and SURVEYREG) available in SAS 9.4 (SAS Institute Inc. 2010) to account for the complex survey design of the MEPS and to obtain weighted parameter estimates and standard errors [ $\left.{ }^{26}\right]$.

\section{Results}

In the baseline year (results not presented in tabular form), majority of adults with diabetes and depression were women $(64.3 \%)$, white $(76.1 \%)$, and lived in metropolitan area (78.2\%). Most adults in our study sample had at least high school education (77.6\%), $28.3 \%$ had income above $400 \%$ of FPL, and $66.9 \%$ had prescription drug coverage. With regard to other chronic physical conditions, the most highly prevalent conditions were hypertension (78.0\%), high cholesterol (64.5\%), and arthritis (52.8\%).

Overall, $59.6 \%$ reported using antidepressants only, $22.4 \%$ reported receiving psychotherapy (with or without antidepressants), and $17.9 \%$ had no depression treatment (Table 1). There were significant subgroup differences in depression treatment. For example, a significantly higher percentage of the younger age group (22-49 years) were treated with psychotherapy (with or without antidepressants) as compared to the elderly over the age of 65 years (29.9 vs. $11.2 \%$ ). In contrast, lower proportion of the younger age group (22-49 years) reported using antidepressants as compared to those over the age of 65 years (50.7 vs. $72 \%)$. A higher proportion of individuals with education above high school education had treated with psychotherapy (with or without antidepressants) in comparison with individuals with less than high school education (28.3 vs. $15.5 \%$ ). Conversely, lower proportion of individuals with above high school education reported using antidepressants compared to individuals with less than high school education (16.6 vs. $25.4 \%$ ).

The mean scores and the standard errors (SE) of the HRQoL measures (PCS and MCS) during the baseline year and the follow-up year are presented in Table 2. During the baseline period, the mean PCS and MCS scores were $37.01(\mathrm{SE}=0.49)$ and $40.78(\mathrm{SE}=0.45)$, 
respectively. During the follow-up year, the mean PCS and MCS scores were 36.79 ( $\mathrm{SE}=$ $0.49)$ and 41.51 (SE = 0.41), respectively (Fig. 1). The established PCS-12v2 and MCS-12v2 norms for the national sample of individuals with depression are 45.55 (SD = 11.71 ) and 37.40 (10.67), respectively. It has to be noted that the normed-based scores of persons with diabetes are $41.52(\mathrm{SD}=11.07)$ and $47.28(\mathrm{SD}=10.72)$ for PCS-12 and MCS-12, respectively $\left[{ }^{24}\right]$.

There were significant differences in the PCS and MCS scores during the follow-up year by the baseline depression treatment categories. Individuals with diabetes and depression who reported using only antidepressants had lower PCS scores as compared to those without any treatment for depression $(-1.19, \mathrm{SE}=0.22, p<0.001)$. However, those who reported receiving psychotherapy with or without antidepressants had higher PCS scores as compared to individuals without depression treatment $(0.49, \mathrm{SE}=0.26, p=0.06)$. With regard to MCS scores, individuals who reported using only antidepressants had higher MCS scores as compared to those without depression treatment $(0.99, \mathrm{SE}=0.08, p<0.001)$. However, those who reported receiving psychotherapy with or without antidepressants had lower MCS scores during the follow-up as compared to those without depression treatment $(-3.76, \mathrm{SE}=$ $0.22, p<0.001)$.

Table 3 displays the regression coefficient estimates and standard errors of depression treatment categories from the separate OLS regressions on PCS and MCS scores. In model 1 , after controlling for the predisposing, enabling, need, external environment characteristics, and personal health practices, the results remained similar to the unadjusted results. However, in model 2, after adding the baseline PCS scores, individuals with diabetes and depression who reported receiving psychotherapy with or without antidepressants had higher PCS scores as compared to those without any treatment for depression (beta $=1.28, p$ $<0.001$ ). Individuals who reported using only antidepressants had lower PCS scores (beta $=$ $-0.54, p<0.001)$ as compared to those without depression treatment.

With regard to the MCS scores, the statistical significance of the associations between depression treatment categories and the MCS scores remained consistent in unadjusted and adjusted models 1 and 2. In model 2, after adding baseline MCS scores, individuals with diabetes and depression who reported receiving psychotherapy with or without antidepressants had lower MCS scores as compared to those without any treatment for depression (beta $=-1.43, p<0.001$ ). Those using only antidepressants had higher MCS scores as compared to those without depression treatment (beta $=0.56, p<0.001$ ).

\section{Discussion}

The current study set out to examine the relationship between depression treatment and HRQoL among adults with diabetes and depression. In our study, $17.9 \%$ of adults with diabetes and depression did not receive antidepressants or psychotherapy for depression. As noted in the introduction, many clinical trials have compared usual care with psychotherapy or collaborative care $\left[{ }^{10}, 13 \_15\right]$. By including "no treatment group," our study was able to capture the relationship between no depression treatment and the HRQoL measures. We are not able to compare our findings with previous literature because previous studies on the 
relationship between depression treatment and chronic disease outcomes have focused on improvement of depression symptoms and the diabetes-specific outcomes as measurements to estimate the effectiveness of depression treatment [9_12]. If our study findings are replicated by other studies, these findings can inform clinical practice about the effectiveness of depression treatment in real-world practice settings among adults with diabetes and depression $[27,28]$.

Compared to the norm for the diabetes population, the average baseline PCS and MCS scores for our study sample was lower, suggesting that our study sample may be sicker. After controlling for predisposing, enabling, need, external environment factors, personal health practices, and baseline HRQoL measures, our findings revealed that psychotherapy (with or without antidepressants) was associated with higher average PCS scores and lower average MCS scores as compared to those without depression treatment after 1-year followup. Individuals who used only antidepressants had lower PCS scores and higher MCS scores as compared to those without depression treatment after 1-year follow-up. These findings suggest that the relationship between depression treatment categories and the HRQoL depends on the type of the HRQoL measure and the type of depression treatment.

We found that individuals using only antidepressant had lower physical HRQoL as compared to those without depression treatment, consistent with one prior study not specific to diabetes $\left[{ }^{29}\right]$. Caruso and colleagues found that among patients with depression and coexisting chronic conditions such as diabetes and hypertension, antidepressant treatment negatively influenced the physical HRQoL $\left[{ }^{29}\right]$. It is plausible that individuals using antidepressants may have poor physical HRQoL because some types of antidepressants have side effects that cannot be tolerated by individuals with diabetes and contraindicated with diabetes $\left[{ }^{30}\right]$. As the sample sizes were very small, we were unable to assess the relationship between the types of antidepressants and the physical HRQoL. Future studies need to examine the association between type of antidepressants and the PCS scores among individuals with diabetes and depression.

We found that antidepressant treatment was associated with higher MCS scores as compared to those without depression treatment, consistent with prior literature $\left[{ }^{29}, 31\right]$. However, these studies did not exclusively focus on individuals with diabetes.

Psychotherapy with and without antidepressants was associated with lower MCS scores during the follow-up. This finding is not consistent with other studies in the general population, which have documented a positive effect of psychotherapy on MCS scores [ $\left.{ }^{32}\right]$. In our study, those with lower MCS scores at baseline were more likely to receive psychotherapy with or without antidepressants. Therefore, it is possible that the severity of depression may be associated with both the receipt of psychotherapy at baseline and its impact on the follow-up MCS scores. As our data did not contain information on severity of depression, we are unable to explain this finding.

The current study had a number of strengths and some limitations. The study sample was derived from a nationally representative survey and included adults with diabetes. We included a comprehensive list of independent variables to assess the relation between 
depression treatment and HRQoL among adults with diabetes and depression. Our study included "no depression treatment" as a comparison group and evaluated the effectiveness of depression treatment in real-world settings.

The limitations of the study need to be considered while interpreting the findings. For example data on all variables were self-reported which could be prone to recall bias. Our study focused on individuals with diagnosed depression and may have missed individuals who do not get treated due to undiagnosed depression. Although we controlled for many covariates that could be associated with HRQoL among adults with diabetes and concurrent depression, we were not able to control for depression severity as well as diabetes severity; these factors may alter the relationship between depression treatment and the HRQoL. We did not differentiate between combined therapy and monotherapy with regard to psychotherapy because we found that a small number of individuals $(N=55)$ reported receipt of psychotherapy who represent only $5.4 \%$ of the study sample. Future research needs to examine the consequences of only psychotherapy intervention on the HRQoL of individuals with diabetes and depression. As the study used a retrospective observational cohort design, causal inferences could not be made. Although included the baseline HRQoL measures in the regression models, OLS regressions may not fully adjust for the baseline differences in HRQoL measures. Furthermore, lack of information on initiation and duration of treatment could have affected the interpretation of the findings.

Despite the limitations, this study addressed the association of depression treatment on HRQoL of adults with diabetes and depression among the non-institutionalized population of the USA using data from a nationally representative survey. Our study findings revealed that the associations between the depression treatment and HRQoL measures varied by the type of depression treatment and the components of the HRQoL.

\section{Acknowledgments}

The authors would like to thank Rini Vohra who shared in revising the early draft of this manuscript. They also thank Dr. Michael Denney for his contributions in revising some parts of the final drafts. Dr. Sambamoorthi is supported by the National Institute of General Medical Sciences, U54GM104942. The content is solely the responsibility of the authors and does not necessarily represent the official views of the NIH or policy or position of West Virginia University (WVU) or any other affiliated organizations.

\section{Abbreviations}

HRQOL

DM

GERD

BMI

RCT
Health-related quality of life

Diabetes mellitus

Gastroesophageal reflux disease

Body mass index

Randomized controlled trial 


\section{References}

1. Pawaskar MD, Anderson RT, Balkrishnan R. Self-reported predictors of depressive symptomatology in an elderly population with type 2 diabetes mellitus: A prospective cohort study. Health Quality Life Outcomes. 2007; 5:50.

2. McCollum M, Ellis SL, Regensteiner JG, Zhang W, Sullivan PW. Minor depression and health status among US adults with diabetes mellitus. The American Journal of Managed Care. 2007; 13(2):6572. [PubMed: 17286526]

3. Egeden L, Nietert P, Zheng D. Depression and allcause and coronary heart disease mortality among adults with and without diabetes. Diabetes Care. 2005; 28(6):1339-1345. [PubMed: 15920049]

4. Kohen D, Burgess AP, Catalán J, Lant A. The role of anxiety and depression in quality of life and symptom reporting in people with diabetes mellitus. Quality of Life Research. 1998; 7:197-204. [PubMed: 9584549]

5. Goldney RD, Phillips PJ, Fisher LJ, Wilson DH. Diabetes, depression, and quality of life: A population study. Diabetes Care. 2004; 27:1066-1070. [PubMed: 15111522]

6. Hanninen JA, Takala JK, Keinanen-Kiukaanniemi SM. Depression in subjects with type 2 diabetes. Predictive factors and relation to quality of life. Diabetes Care. 1999; 22:997-998. [PubMed: 10372255]

7. Eren I, Erdi O, Sahin M. The effect of depression on quality of life of patients with type II diabetes mellitus. Depression and Anxiety. 2008; 25:98-106. [PubMed: 17311266]

8. Egede LE, Ellis C. The effects of depression on metabolic control and quality of life in indigent patients with type 2 diabetes. Diabetes Technology \& Therapeutics. 2010; 12(4):257-262. [PubMed: 20210563]

9. Williams JW Jr, Katon W, Lin EH, Nöel PH, Worchel J, Cornell J, et al. The effectiveness of depression care management on diabetes-related outcomes in older patients. Annals of Internal Medicine. 2004; 140(12):1015-1024. [PubMed: 15197019]

10. Katon WJ, Von Korff M, Lin EH, Simon G, Ludman E, Russo J, et al. The pathways study: A randomized trial of collaborative care in patients with diabetes and depression. Archives of General Psychiatry. 2004; 61:1042-1049. [PubMed: 15466678]

11. Paile-Hyvärinen M, Wahlbeck K, Eriksson JG. Quality of life and metabolic status in mildly depressed patients with type 2 diabetes treated with paroxetine: A double-blind randomized placebo controlled 6-month trial. BMC Family Practice. 2007; 8:34. [PubMed: 17570858]

12. Echeverry D, Duran P, Bonds C, Lee M, Davidson MB. Effect of pharmacological treatment of depression on $\mathrm{A} 1 \mathrm{C}$ and quality of life in low-income Hispanics and African Americans with diabetes: A randomized, double-blind, placebocontrolled trial. Diabetes Care. 2009; 32(12):21562160. [PubMed: 19729522]

13. Lam RW, Parikh SV, Ramasubbu R, Michalak EE, Tam EM, Axler A, et al. Effects of combined pharmacotherapy and psychotherapy for improving work functioning in major depressive disorder. British Journal of Psychiatry. 2013; 203(5):358-365. [PubMed: 24029535]

14. Wu B, Jin H, Vidyanti I, Lee P, Ell K, Wu S. Collaborative depression care among Latino patients in diabetes disease management, Los Angeles, 2011-2013. Preventing Chronic Disease. 2014; 11:140081.

15. Ell K, Katon W, Xie B, Lee PJ, Kapetanovic S, Guterman J, et al. Collaborative care management of major depression among low-income, predominantly Hispanic subjects with diabetes: A randomized controlled trial. Diabetes Care. 2010; 33(4):706-713. [PubMed: 20097780]

16. Andersen RM. Revisiting the behavioral model and access to medical care: Does it matter? Journal of Health and Social Behavior. 1995; 36:1-10. [PubMed: 7738325]

17. Cohen SB, DiGaetano R, Goksel H. Methodology Report\# 5: Estimation procedures in the 1996 MEPS household component. 1999

18. Smith M, Davis MA, Stano M, Whedon JM. Aging baby boomers and the rising cost of chronic back pain: Secular trend analysis of longitudinal Medical Expenditures Panel Survey data for years 2000 to 2007. Journal of Manipulative and Physiological Therapeutics. 2013; 36(1):2-11.

[PubMed: 23380209] 
19. Machlin SR, Chevan J, Yu WW, Zodet MW. Determinants of utilization and expenditures for episodes of ambulatory physical therapy among adults. Physical Therapy. 2011; 91(7):1018-1029. [PubMed: 21566066]

20. Medical Expenditure Panel Survey, Household Component. http://meps.ahrq.gov/mepsweb/ survey_comp/household.jsp. Accessed 9 March 2014.

21. Medical Expenditure Panel Survey Clinical Classification Code to ICD-9-CM Code Crosswalk. http://meps.ahrq.gov/mepsweb/data_stats/download_data/pufs/h128/h128_icd9codes.shtml. Accessed 11 March 2014.

22. Lobo IE, Loeb DF, Ghushchyan V, Schauer IE, Huebschmann AG. Missed opportunities for providing low-fat dietary advice to people with diabetes. Preventing Chronic Disease. 2012; 9:E161. [PubMed: 23116780]

23. Ware JE, Kosinski M, Keller SD. A 12-item short-form health survey construction of scales and preliminary tests of reliability and validity. Medical Care. 1996; 34:220-233. [PubMed: 8628042]

24. Ware, JE.; Kosinski, M.; Turner-Bowker, DM.; Gandek, B. SF-12v2: how to score version 2 of the SF-12 health survey (with a supplement documenting version 1). Lincoln, RI: QualityMetric Inc; 2002.

25. Fortney JC, Harman JS, Xu S, Dong F. The association between rural residence and the use, type, and quality of depression care. The Journal of Rural Health. 2010; 26(3):205-213. [PubMed: 20633088]

26. Bharmal M, Thomas J. Health insurance coverage and health-related quality of life: analysis of 2000 Medical Expenditure Panel Survey data. Journal of Health Care for the Poor and Underserved. 2005; 16(4):643-654. [PubMed: 16311490]

27. Xie J, Wu EQ, Zheng ZJ, Sullivan PW, Zhan L, Labarthe DR. Patient-reported health status in coronary heart disease in the United States: Age, sex, racial, and ethnic differences. Circulation. 2008; 118(5):491-497. [PubMed: 18625894]

28. Moriarty DG, Zack MM, Kobau R. The Centers for Disease Control and Prevention's Healthy Days Measures - population tracking of perceived physical and mental health over time. Health and Quality of Life Outcomes. 2003; 2(1):37. [PubMed: 14498988]

29. Caruso R, Rossi A, Barraco A, Quail D, Grassi L. The Factors Influencing Depression Endpoints Research (FINDER) study: Final results of Italian patients with depression. Annals of General Psychiatry. 2010; 9(1):33. [PubMed: 20670396]

30. Goodnick PJ, Henry JH, Buki V. Treatment of depression in patients with diabetes mellitus. Journal of Clinical Psychiatry. 1995; 56(4):128-136. [PubMed: 7713850]

31. O’Neil A, Sanderson K, Oldenburg B, Taylor CB. Impact of depression treatment on mental and physical health-related quality of life of cardiac patients: A meta-analysis. Journal of Cardiopulmonary Rehabilitation and Prevention. 2011; 31(3):146-156. [PubMed: 21150634]

32. Matsunaga M, Okamoto Y, Suzuki SI, Kinoshita A, Yoshimura S, Yoshino A, et al. Psychosocial functioning in patients with treatment-resistant depression after group cognitive behavioral therapy. BMC Psychiatry. 2010; 10(1):22. [PubMed: 20230649] 


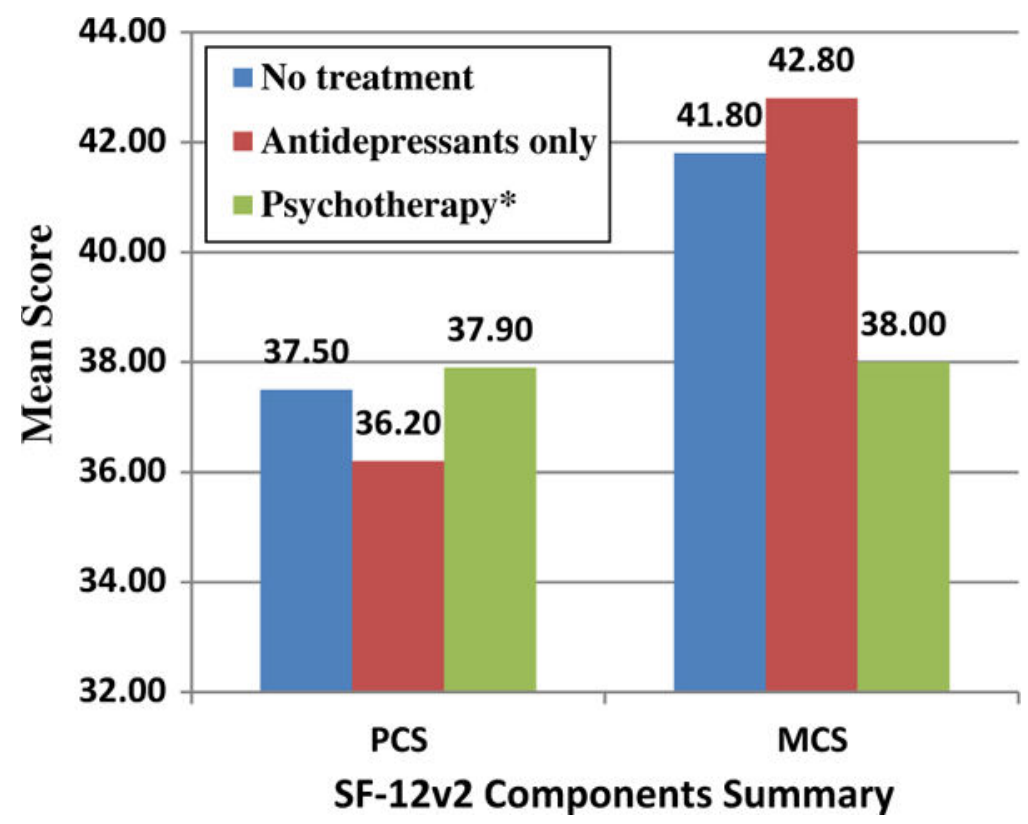

Fig. 1.

Follow-up mean physical and mental component summary scores by depression treatment categories. Asterisk Psychotherapy with or without antidepressants use; PCS physical component summary Short Form Health Survey SF-12 version 2, MCS mental component summary Short Form Health Survey SF-12 version 2 


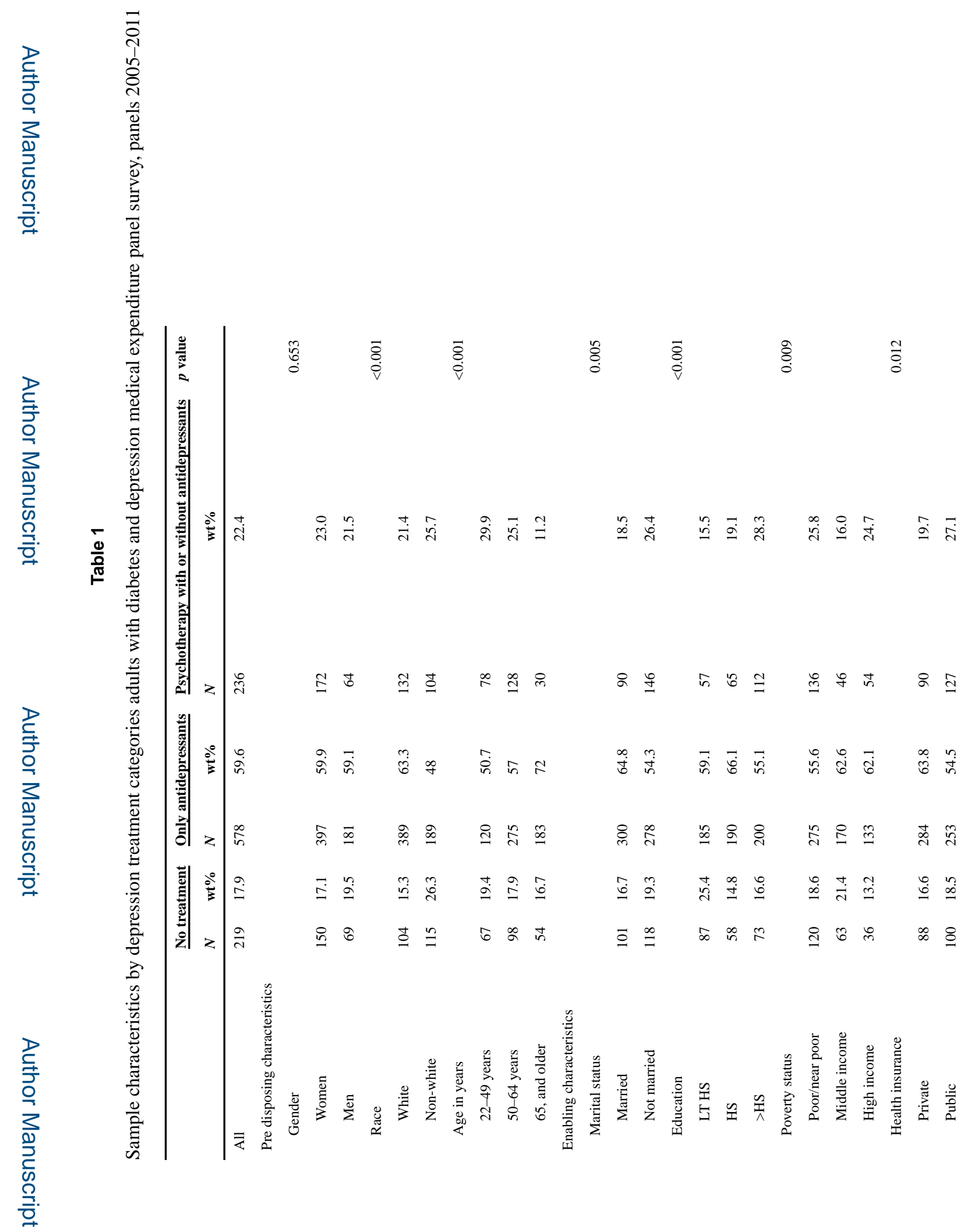

Qual Life Res. Author manuscript; available in PMC 2017 June 01. 

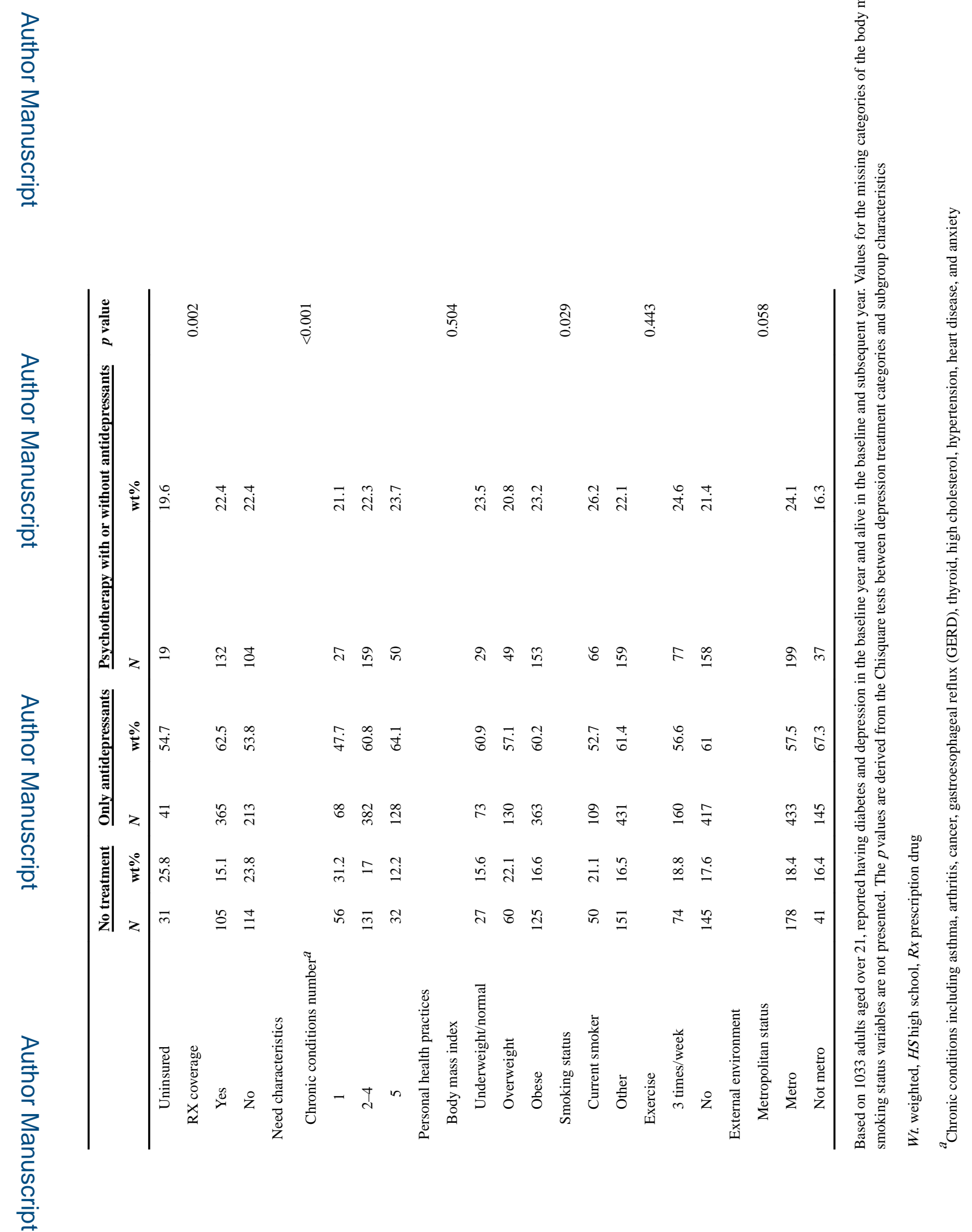

Qual Life Res. Author manuscript; available in PMC 2017 June 01. 


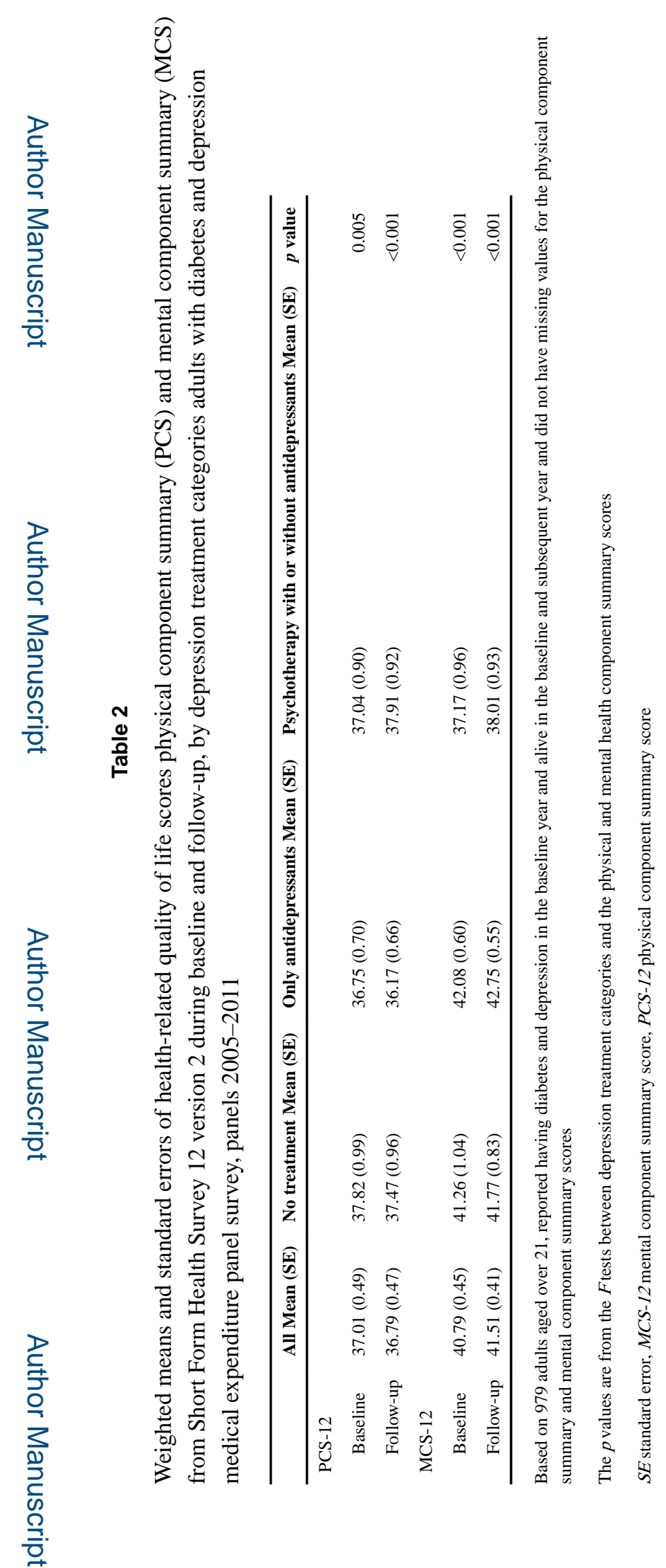

Qual Life Res. Author manuscript; available in PMC 2017 June 01. 


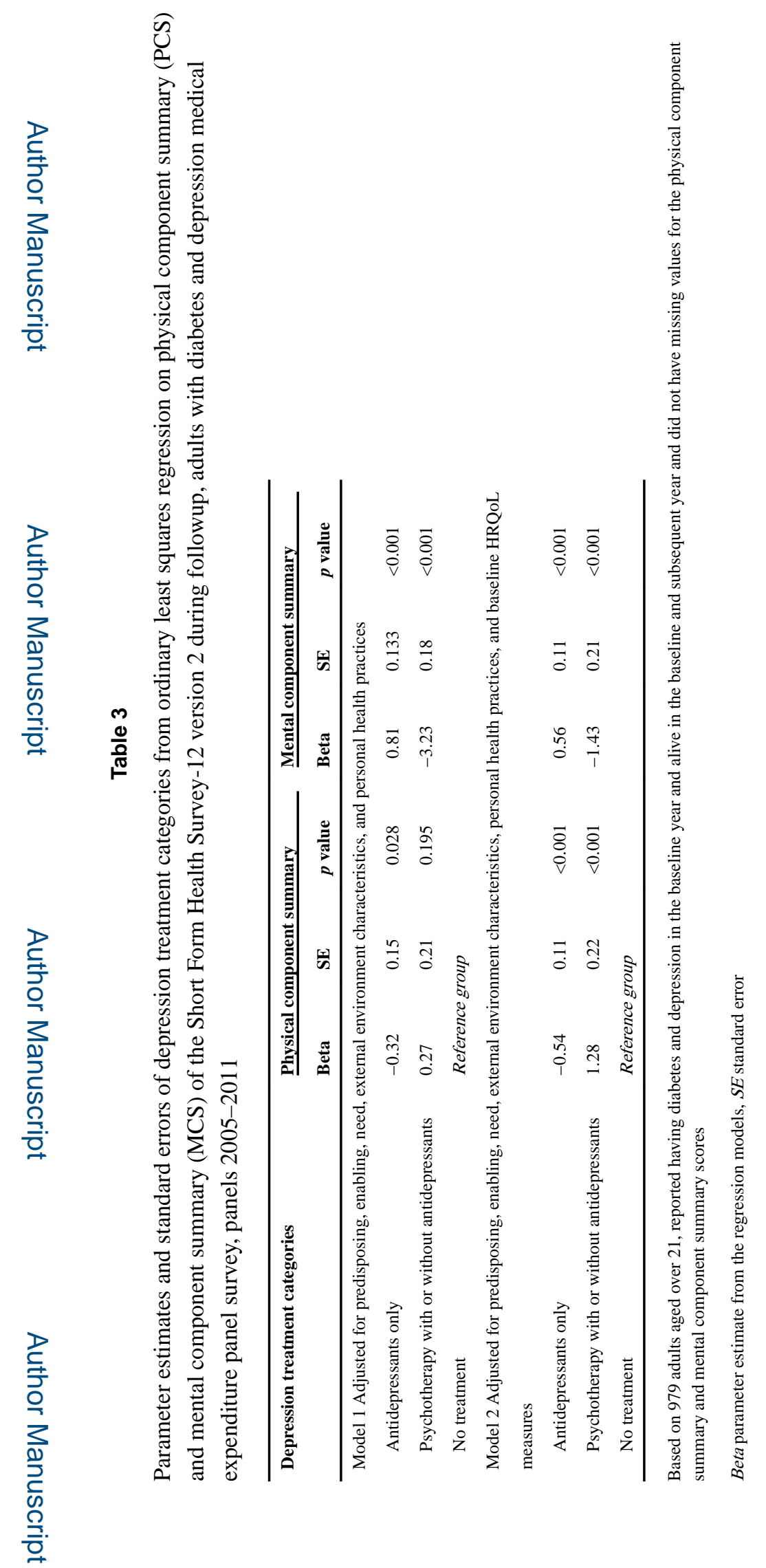

Qual Life Res. Author manuscript; available in PMC 2017 June 01. 